\title{
E2A suppresses invasion and migration by targeting YAP in colorectal cancer cells
}

\author{
Hongchao Zhao ${ }^{1,2+}$, Ao Huang ${ }^{1,2+}$, Pu Li ${ }^{1 \dagger}$, Yingjun Quan ${ }^{1,2}$, Bo Feng ${ }^{1,2}$, Xuehua Chen ${ }^{1}$, Zhihai Mao ${ }^{1,2^{*}}$, \\ Zhenggang Zhu ${ }^{1}$ and Minhua Zheng ${ }^{1,2^{*}}$
}

\begin{abstract}
Background: E2A gene, which encodes two basic helix-loop-helix (bHLH) transcription factors E12 and E47, has been identified as regulator of B lymphoid hematopoiesis and suppressor of lymphoma. E47 protein was found to decrease E-cadherin expression and induce epithelial-mesenchymal transition (EMT). However, the role of E2A in colorectal cancer (CRC) metastasis is still elusive.

Methods: qRT-PCR and semi-qRT-PCR were performed to determine mRNA level of E2A in CRC specimens and colorectal cancer cells. RNAi was employed to downregulate E2A expression and subsequent protein level change was evaluated by immunoblot. Cell invasion and migration capacity were detected by transwell assay using cell culture inserts with or without basement membrane matrix, respectively.

Results: E2A expression was decreased in metastatic CRC tissues. Invasion and migration assays showed downregulation of E2A increased metastatic capacity of CRC cells while forced expression of E12 or E47 could offset this effect. Both E12 and E47 suppressed EMT induced by E2A downregulation. Moreover, Yes-Associated Protein (YAP) was a downstream target of E2A and suppression of YAP inhibited the pro-migration/invasion of E2A deficiency.
\end{abstract}

Conclusion: Our results suggest that E2A suppresses CRC cell metastasis, at least partially if not all, by inhibiting YAP expression.

Keywords: Metastasis, E2A, Colorectal cancer, YAP, EMT

\section{Background}

Colorectal cancer (CRC) is one of the most prevalent malignancies worldwide, ranking the third of all cancerrelated deaths, and distant metastasis is the major cause of deaths for CRC patients [1]. These secondary tumors arise as the result of a multi-step process which begins when cancer cells within primary tumors break away from the microenvironment and invade through the basement membrane [2]. Although many metastasis-related genes have been identified in CRC [3], the complicated molecular mechanism of CRC development and progression is not yet fully understood.

\footnotetext{
* Correspondence: zhihai.mao@gmail.com; davisd0zhao@hotmail.com ${ }^{\dagger}$ Equal contributors

'Department of Surgery, Shanghai Key Laboratory of Gastric Neoplasms, Shanghai Institute of Digestive Surgery, Ruijin Hospital, School of Medicine, Shanghai Jiao Tong University, 197 Rui Jin Er Rd, Shanghai 200025, People's Republic of China

${ }^{2}$ Shanghai Minimally Invasive Surgery Center, Shanghai, China
}

The E2A gene encodes two basic helix-loop-helix (bHLH) transcription factors, E12 and E47, by means of variant splicing [4]. The E2A proteins (E12/E47) belong to the class I bHLH family and regulate expression of target genes by binding DNA with tissue-specific Class II HLH proteins, either as homodimers or as heterodimers [5-7]. Previous studies have shown that E12/E47 proteins are distributed widely in most adult tissues although at different expression levels [8,9]. Both proteins are indispensable for some normal tissue development while deficiency or aberrant expression of E2A could lead to tumorigenesis. For instance, E12/E47 proteins are required for B-cell differentiation and Ig gene rearrangements [10]; two aberrant fusion proteins, E2A-HLF and E2A-PBX1, lead to pro-B cell acute lymphoblastic leukemia (ALL) and pre-B cell ALL [11]. In addition, targeted disruption of the E2A gene leads to thymic lymphomas, suggesting that E2A gene products can act as tumor 
suppressors $[12,13]$. Specifically, the expression of epithelial maker E-cadherin was repressed upon E12/E47 binding to the E-pal element, a palindromic sequence between -75 and -86 in the E-cadherin promoter [14].

In colorectal cancer, ectopic expression of the E47 efficiently sequestered endogenous Id proteins from Id-bHLH heterodimers, leading to growth arrest of the cells [15]. However, it still remains unknown whether E12/E47 proteins affect CRC cells metastasis or not. In the present study, we demonstrated that E2A down-regulation was required for the development of metastasis while ectopic expression of E2A in CRC cells could suppress invasion and migration in vitro. Furthermore, we identified, and validated YAP (Yes-Associated Protein) as a novel downstream target of E2A.

\section{Methods}

\section{Cell culture}

Human colorectal cancer cell lines, SW480 and Caco-2, were purchased from American Type Culture Collection (ATCC). SW480 was cultured in Leibovitz' L-15 Medium (Corning Cellgro ${ }^{\circ}$, Manassas, VA, USA) with $10 \%$ fetal bovine serum (FBS) (Invitrogen, Carlsbad, CA, USA), and Caco-2 in Minimum Essential Medium (Corning Cellgro $\left.{ }^{\circ}\right)$ with $20 \% \mathrm{FBS}$. Cells were maintained at $37^{\circ} \mathrm{C} / 5 \% \mathrm{CO}_{2}$ in a humidified incubator.

\section{Clinical specimens}

The clinical research protocol was approved by the Ethical Committee of Ruijin Hospital, Shanghai Jiao Tong University School of Medicine. Seventy-five surgical specimens of primary CRC tumors were obtained from Shanghai Minimal Invasive Surgery Center (2012-2013) with written informed consents given by all patients prior to surgery. Inclusion criteria were: without neoadjuvant chemoradiotherapy; resectable colorectal cancers; without evidences of primary tumors of other organs; able to be interviewed during the follow-up. Fresh tumor tissues were harvested immediately after samples' dissection, snap-frozen in liquid nitrogen, and preserved at $-80^{\circ} \mathrm{C}$. The pathologic classification/staging of tumors was performed in accordance to the Cancer Staging Manual from the International Union Against Cancer ( $7^{\text {th }}$ edition, 2009). Tumor samples were classified into two groups: tumors with metastatic sites and tumors without metastases, based on the status of lymph node and/or distant metastasis.

\section{Protein extraction and immunoblot}

RIPA buffer (Solarbio, Beijing, China) containing protease inhibitor cocktail (Roche Applied Science, Basel, Switzerland) was used to extract total tissue and cell proteins, according to manufacturer's instruction. Immunoblot was done according to the standard protocol, with primary antibodies against E2A (Santa Cruz, Dallas, Texas, USA), vimentin (Santa Cruz), E-cadherin (Cell Signaling Technol., Danvers, MA, USA), $\beta$-catenin (Cell Signaling Technol.), YAP (Cell Signaling Technol.) and MMP-9 (Cell Signaling Technol.). Goat anti-mouse or goat antirabbit IgG conjugated to horseradish peroxidase (HRP, Pierce, Rockford, IL, USA) was used as the secondary antibody. Chemiluminescent signals were visualized using SuperSignal West Pico Chemiluminescent Substrate (Pierce) and the signal intensity was analyzed using the Image Lab ${ }^{\text {Tw }}$ Software Version 4.0.1 (BIO-RAD, Hercules, CA, USA). The experiments were performed in triplicate with GAPDH (Kangchen, Shanghai, China) as endogenous control.

\section{Immunofluorescence}

Cells growing on slides (Millipore, Billerica, MA, USA) were fixed with $4 \%$ paraformaldehyde (PFA, SigmaAldrich, St.Louis, USA) and permeabilized for $10 \mathrm{~min}$ using $0.1 \%$ TritonX-100/phosphate buffered saline (PBS). Non-specific antigens were then blocked with $3 \%$ BSA in PBS-T $(0.2 \%$ Tween 20$)$ for $1 \mathrm{~h}$ at room temperature. After washing, cells were incubated with primary antibodies against vimentin (Santa Cruz), E-cadherin (Cell Signaling Technol.), $\beta$-catenin (Cell Signaling Technol.) in $4^{\circ} \mathrm{C}$ overnight, followed by incubation with Alex Fluor 555 anti-Rabbit IgG (Cell Signaling Technol.) for 2 hours at room temperature. Images were examined and captured using an Olympus Fluoview Confocal Microscope. Rabbit mAb IgG XP isotype (Cell Signaling Technol.) was used as negative control.

\section{Invasion and migration assay}

Cell invasion and migration assays were performed using cell culture inserts (Millipore) coated with or without basement membrane matrix (BD Bioscience, Bedford, MA, USA), respectively. Assays were performed as previously described [16,17]. In brief, approximate $1 \times 10^{5}$ cells resuspended in $200 \mu \mathrm{l}$ non-serum culture medium were placed triplicatedly in upper chamber of insert and medium with $10 \%$ FBS was used as chemo-attractant in lower chamber; inserts were incubated at $37^{\circ} \mathrm{C}$ for 48 hours in a $5 \% \mathrm{CO}_{2}$ humidified incubator. Cells attached on the inner side of the chamber were then cleared softly with cotton swab and cells outside the insert were stained in $1 \%$ crystal violet for 30 minutes. Cells in five random fields were counted under microscope and the relative invasion and migration capacity were interpreted as the average number of cells \pm SD per field.

\section{RNA extraction and RT-PCR}

Total RNAs from cell lines and tissues were extracted using Trizol reagent (Invitrogen) according to the manufacturer's 
instruction. Reverse transcription of RNAs was performed using GoScript ${ }^{\mathrm{TM}}$ Reverse Transcriptase System (Promega, Madison, WI, USA) as per protocol. The mRNA level of YAP (forward: 5' - TAGCCC TGCGT AGCCA GTTA -3'; reverse: $5^{\prime}$ - TCATGC TTAGT CCACTGTCTGT -3') in cell lines was assessed by semi-qRT-PCR using Taq PCR MasterMix (Tiangen, Beijing, China). GAPDH (forward: 5'-GGAGC GAGAT CCCTC CAAAAT-3'; reverse: 5' GGCTG TTGTC ATACT TCTCA TGG-3') was used as an internal loading control. The expression of E2A (forward: 5' - CCACTT CACTG AGTCGC ACAG -3'; reverse: 5' - GTCTCT CCCGAA GGAGG CATA -3') and YAP in tumor tissues were detected by real-time qRTPCR using SYBR Green PCR Master Mix (Invitrogen) on the Applied Biosystems 7900HT sequence detection system with GAPDH as endogenous control.

\section{Transient transfection}

Short hairpin RNA (shRNA) against human YAP and shRNA negative control (shNC) were bought from GenePharma (Shanghai, China). Plasmids pEZ-M29-E12 and pEZ-M29-E47, encoding fusion protein of eGFP-E12 or eGFP-E47 respectively, were purchased from Genecopoeia (Rockville, MD, USA). Cells were seeded in 6-well culture plates one day before transient transfection, which was performed with lipofectamine 2000 (Invitrogen), according to the instruction of manufacturer. Forty-eight hours after transfection, cells were harvested and the protein levels of the targeted genes were assessed by immunoblot, with GAPDH as loading control.

\section{Lentiviral transfection for stable expression clones}

Plasmids $\mathrm{pL} / \mathrm{shRNA} / \mathrm{F}-\mathrm{shR}$ with shE2A or shNC (negative control), namely LV-shE2A and LV-shNC, were purchased from Novobio (Shanghai, China). Lentivirus transfection was performed according to the manufacturer's instruction to establish shE2A-expressing stable clones in SW480 cells (SW480/shE2A). The control clone (SW480/shNC) was constructed similarly. E2A protein expressions of abovementioned clones were examined by immunoblot using GAPDH as loading control.

\section{Statistical analysis}

Two-tailed Student's $t$-test, Spearman's correlation or one-way ANOVA were used for statistical analysis when appropriate. All statistical analyses were performed using the SPSS 16.0 (SPSS Inc., Chicago, IL, USA). A twotailed value of $\mathrm{p}<0.05$ was considered statistically significant.

\section{Results}

Expression of E2A was decreased in metastatic CRCs

To determine the role of E2A in CRC metastasis, we evaluated the mRNA expression level of E2A in 75 clinical specimens using qRT-PCR. Of the 75 cases, 43 patients were male and 32 were female with a median age of 56 years (range: 41-76 years); besides, 41 cases were metastasis negative and 34 were positive (including pathology confirmed lymph node and CT-scan detected distant metastases). As shown in Figure 1A, E2A mRNA expression was significantly decreased in tumors with metastases compared to those without $(p<0.01)$. We then made a correlation analysis to detect the relationship between E2A expression and clinicalpathological variables by classifying patients into E2A low or high group using the median E2A expression level as cutoff value. As shown in Table 1, expression of E2A was not related to gender $(p=0.653)$, age $(p=0.272)$, tumor histology $(p=0.410)$, or tumor site $(p=0.874)$, but inversely associated with advanced TNM stage $(p=0.010)$.

To further confirm that E2A was also down-regulated at protein level in tumors with metastases, immunoblot was performed using 6 metastatic and 6 non-metastatic tumors chosen randomly from each group. As demonstrated in Figure 1B, metastatic tumors showed lower expression level of E2A protein. Taken together, lower E2A expression associates with positive metastatic status in CRCs.

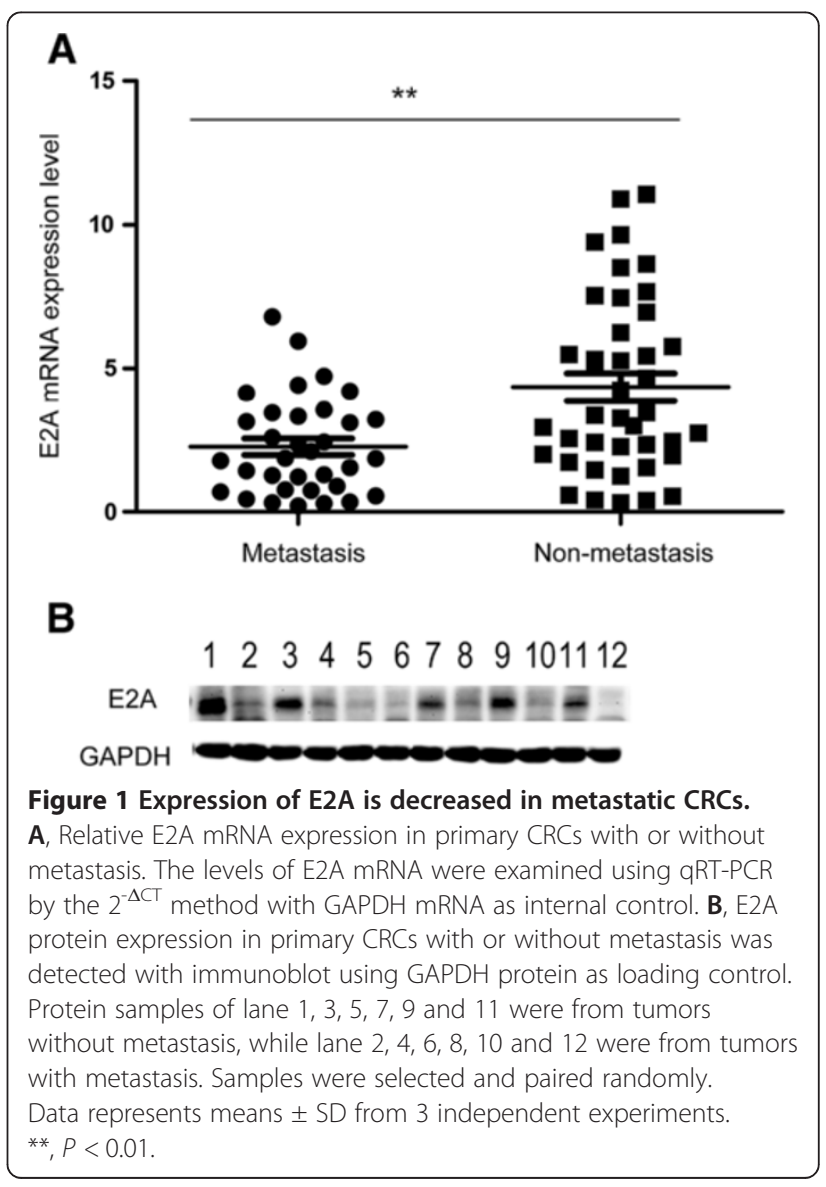


Table 1 Relationship between E2A expression level and clinicopathologic variables in CRC patients

\begin{tabular}{|c|c|c|c|c|}
\hline \multirow[t]{2}{*}{ Variables } & \multirow[t]{2}{*}{ Subgroup } & \multicolumn{2}{|c|}{ E2A expression } & \multirow[t]{2}{*}{$P$ value } \\
\hline & & Low & High & \\
\hline \multirow[t]{2}{*}{ Gender } & Male & 26 & 17 & 0.653 \\
\hline & Female & 21 & 11 & \\
\hline \multirow[t]{2}{*}{ Age (years) } & $<60$ & 22 & 15 & 0.272 \\
\hline & $\geqq 60$ & 25 & 13 & \\
\hline \multirow[t]{2}{*}{ Tumor histology } & Tubular & 39 & 21 & 0.410 \\
\hline & Mucinous & 8 & 7 & \\
\hline \multirow[t]{3}{*}{ Tumor site } & Rectum and sigmoid & 31 & 18 & 0.874 \\
\hline & Right colon & 11 & 7 & \\
\hline & Left colon & 5 & 3 & \\
\hline \multirow[t]{4}{*}{ TNM stage } & 1 & 5 & 7 & 0.010 \\
\hline & $\|$ & 16 & 13 & \\
\hline & III & 18 & 7 & \\
\hline & IV & 8 & 1 & \\
\hline \multirow[t]{2}{*}{ Metastasis } & Negative & 21 & 20 & 0.024 \\
\hline & Positive & 26 & 8 & \\
\hline
\end{tabular}

E2A suppressed CRC cells invasion and migration

Next we wanted to know whether E2A was involved in regulation of CRC metastasis. To this end, SW480 cells were transfected with LV-shE2A to establish SW480/ shE2A stable clones and LV-shNC was used as control. Transfection efficacy was verified by immunoblot and qRT-PCR (Figure 2A). Then we conducted cell invasion and migration assays. As shown in Figure 2B, downregulation of E2A increased the invasion and migration ability of SW480 cells by $\sim 1.2$ folds compared with the blank and shNC groups $(p<0.01)$. Given that E2A has two transcriptional variants E12 and E47, we went a step further by transiently transfecting SW480/shE2A cells with either pEZ-M29-E12 or pEZ-M29-E47 to ectopically express E12 or E47 to discover the isoform responsible for the suppression effect. The transfection efficacy was validated by immunoblot and qRT-PCR (Figure 2C). As demonstrated in Figure 2D, both E12 and E47 reduced invasion and migration of SW480/shE2A cells $(p<0.01)$; importantly, no significant differences in suppression effect between E12 and E47 were observed.

Then we used another colorectal cancer cell line, Caco-2, to investigate whether E2A exerted its function

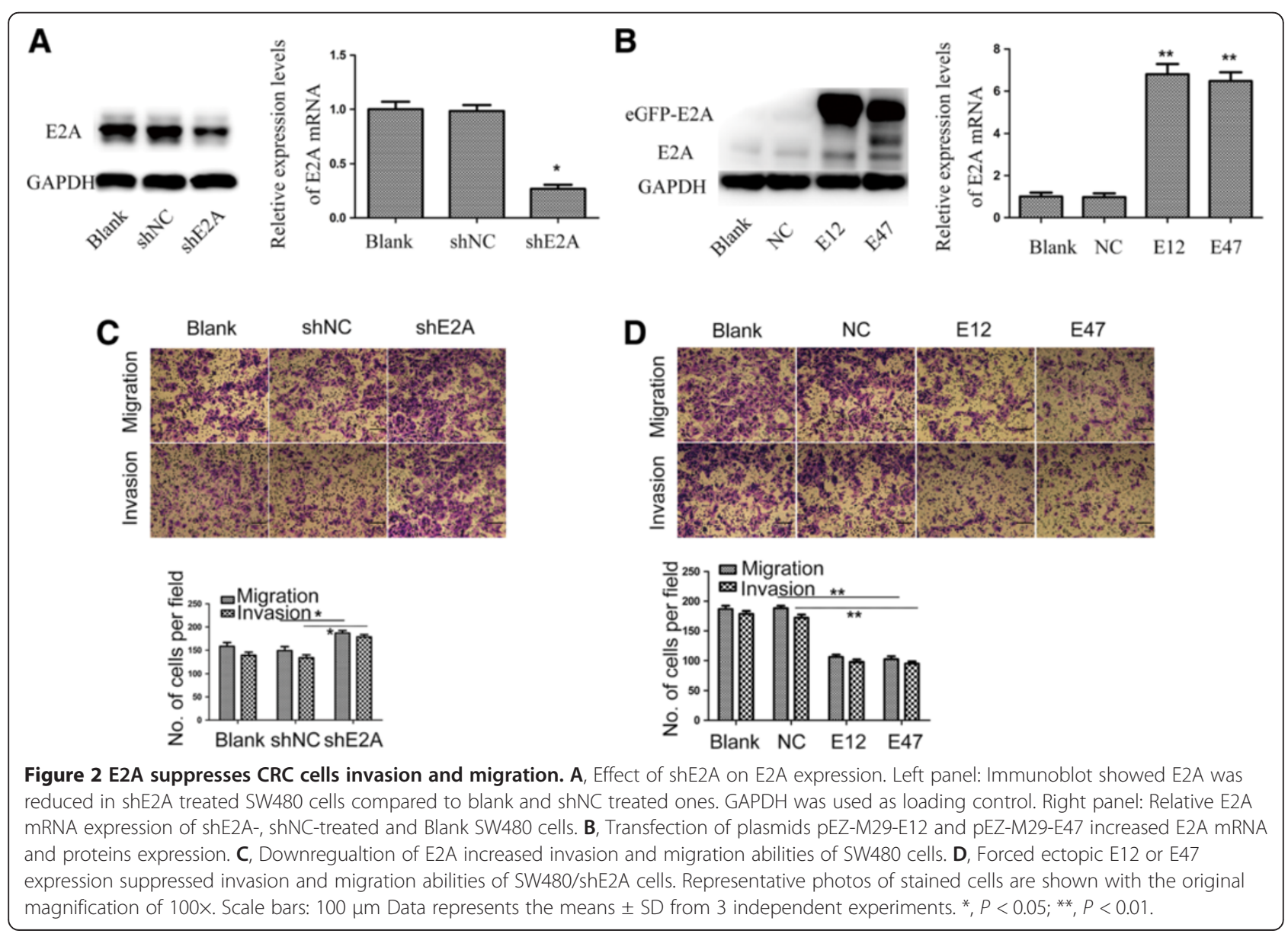


in a cell line specific manner. Similarly, we constructed two stable clones, Caco-2/shE2A (expressing shE2A) and Caco-2/shNC (expressing negative control) and as observed in SW480 cells, metastasis ability of Caco-2 cells increased upon shE2A transfection and was suppressed by E12 and E47 (Additional file 1: Figure S1A \& B), suggesting the metastasis suppression effect of E2A was not cell line dependent. Hence, E2A was a metastasis suppressor gene in CRC.

\section{E2A inhibited the EMT program}

In recent years, EMT has gained more attentions due to its importance in the acquisition metastatic potential during cancer progression [18-20]. Given the fact that E2A was decreased in metastatic CRCs and knockdown of E2A in CRC cells could promote invasion and migration, we wanted to know whether E2A could regulate
EMT program in CRC cells. Indeed, expression of the epithelial marker E-cadherin was decreased and the mesenchymal markers vimentin and $\beta$-catenin were increased in SW480/shE2A cells (Figure 3A). In consistent with increased invasion ability, the expression of matrix metalloproteinases 9 (MMP-9) was elevated after downregulation of E2A (Figure 3A). Similarly, we transfected E12 and E47 plasmids separately into SW480/shE2A cells to identify which one was responsible for EMT regulation. As shown in Figure 3B, both E12 and E47 suppressed the transition induced by shE2A, with vimentin and $\beta$-catenin both reduced about fifty percent and E-cadherin enhanced by $\sim 2$ folds. Moreover, expression of these EMT makers didn't show significant differences between E12- and E47-transfected SW480/shE2A cells $(p>0.05)$. Also, MMP-9 decreased after E12 and E47 transfection (Figure 3B).

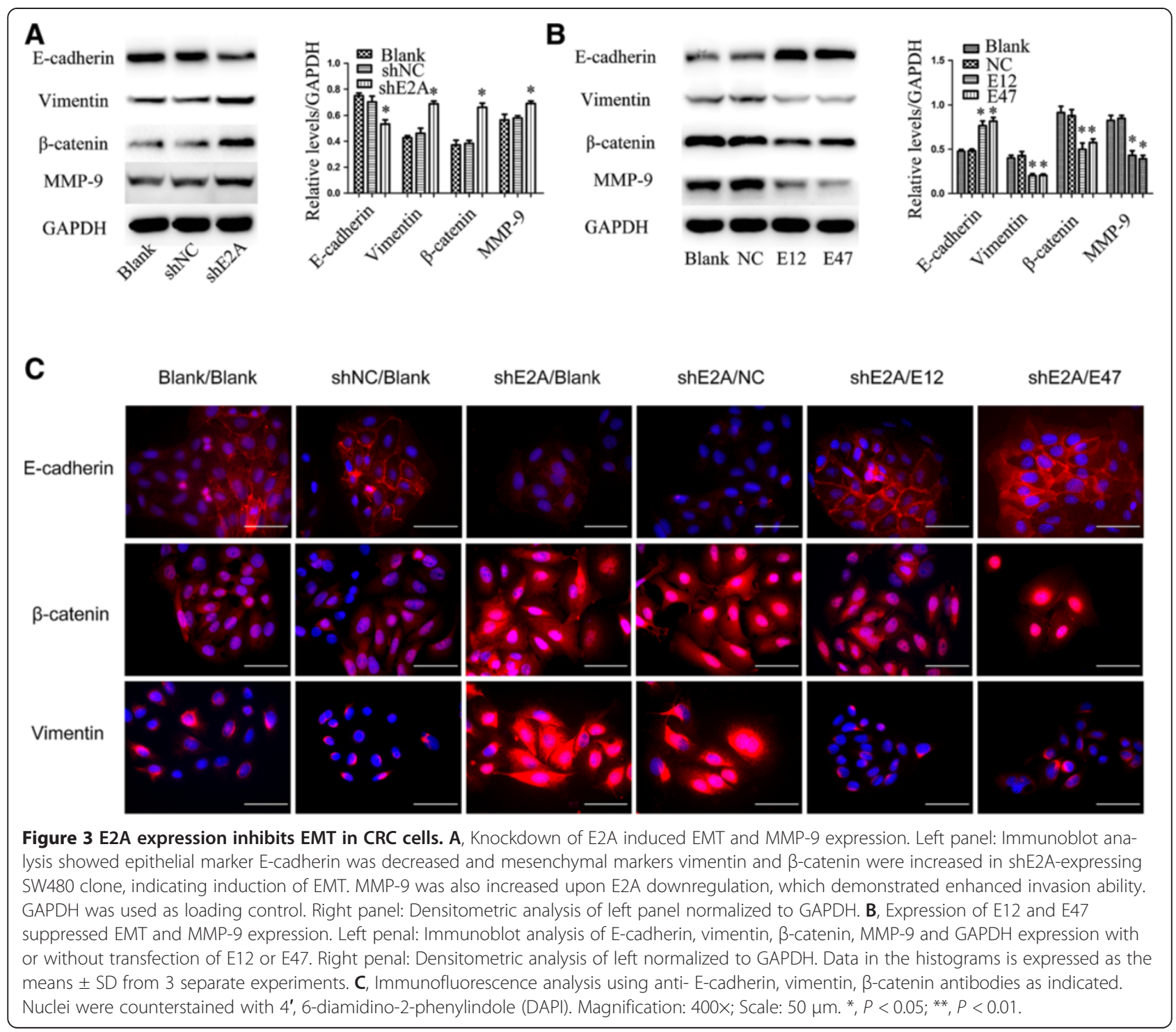


To further demonstrate the role of E2A in EMT program regulation, we performed immunofluorescence to visualize these EMT markers in transfected SW480 cells. In coincidence with immunoblot results, immunofluorescence showed that E-cadherin was significantly decreased while vimentin and $\beta$-catenin were increased in SW480/shE2A cells compared with SW480 and SW480/ shNC cells (Figure 3C). Furthermore, both E12 and E47 restored the expression of E-cadherin in SW480/shE2A cells while suppressed vimentin and $\beta$-catenin (Figure 3C). Thus, E2A may suppress invasion and migration through inhibiting EMT in CRC.

\section{YAP was a downstream target through which E2A suppressed metastasis}

The findings above further led us to explore the potential molecules with which E2A interacted to regulate metastasis in CRC. As we described later, YAP (Yes-Associated Protein) was found to be one downstream target. We detected YAP mRNA expression in CRC tissues and found that YAP was inversely correlated with expression of E2A mRNA $(r=0.491, p<0.01$, Additional file 2: Figure S2), indicating YAP might be modulated by E2A in a suppressive manner. To find whether YAP was regulated by E2A, semi-qRT-RCR and immunoblot were performed to detect the expression of YAP mRNA and protein level after shE2A, E12 and E47 transfection. As shown in Figure 4A and 4B, YAP expression was increased both at $\mathrm{mRNA}$ and protein level in SW480/shE2A cells compared with control cells. Accordingly, both E12 and E47 plasmids attenuated shE2A-induced increase of YAP expression. Taken together, YAP was regulated by E2A.

Next, we asked whether the increased YAP in SW480/ shE2A cells led to the enhanced cell aggressiveness. To this end, transient transfection of shYAP was performed in SW480/shE2A cells to down-regulate YAP (Additional file 3: Figure S3). As shown in Figure 4C, compared to cells transfected with negative control or blank cells, the invasion and migration ability of SW480/shE2A cells was significantly reduced by shYAP to the similar levels as observed in SW480/shNC cells. This finding suggested that the enhanced YAP by shE2A in SW480 cells was critical in the regulation of cell invasion and migration. Besides, downregulation of YAP impaired invasion and migration capacity of SW480 cells (Figure 4D). More importantly, MMP-9 expression was reduced to $50 \%$ of its normal level after shYAP transfection and changes of EMT markers, i.e. increased expression of E-cadherin and decreased vimentin, suggested a suppression of this program (Figure 4E). Immunoblot and immunofluorescence confirmed the expression alterations of E-cadherin and vimentin after shYAP transfection in SW480 cells (Figure 4E \& F). Conclusively, YAP was a target through which E2A regulated EMT program to suppress invasion and migration in CRC cells.

\section{Discussion}

Colorectal carcinogenesis is a multistep process mediated by complex cascades of molecular events governing genomic stability and cell proliferation [21]. Distant metastases, rather than the primary tumors from which these lesions arise, are responsible for $>90 \%$ of carcinomaassociated mortality [22]. In the present study, we demonstrated the suppressive role of E2A in colorectal cancer cell invasion and migration; furthermore, YAP was demonstrated to be a downstream target of E2A in the metastasis of CRC cells.

E2A has been well described as a regulator of early B cell development, and it was dysregulated in lymphoma and breast cancer [13,23]. Decreased expression of E2A has been reported in metastatic pancreatic cancer cell lines [24]. In colorectal adenocarcinomas, ectopic expression of E47 results in proliferation inhibition [15]. The expression of E2A in CRCs is unknown and its role in CRC metastasis is also elusive. In this study, for the first time we investigated the association between E2A expression and CRC metastasis status and we found E2A was decreased in CRCs with metastases both at mRNA and protein levels, indicating its negative relation to $\mathrm{CRC}$ progression. In vitro invasion and migration assays also supported this: downregulation of E2A enhanced metastatic capacity of CRC cells while E12 and E47 could offset this effect. Taken together, E2A has a metastasis suppressive role in CRC. Additionally we found E2A may exert its action by regulating EMT. The EMT program plays an important role in tumor progression and metastasis $[18,25]$. Loss of epithelial traits and gain of mesenchymal features make epithelial tumor cells undergo morphological changes and acquire enhanced metastatic abilities. In our study, we found E2A downregulation inhibited the expression of epithelial marker E-cadherin and increased mesenchymal markers vimentin and $\beta$-catenin in SW480 cells, indicating EMT suppression by E2A. Considering that E-cadherin was regulated by multiple signal pathways [26], we speculate enhanced $\beta$-catenin expression was the main reason for decreased E-cadherin. However, the definite role of E2A in EMT regulation remains further study.

In further investigating the mechanism of action of E2A, we found YAP was regulated as a downstream target. The YAP gene is located on chromosome 11q22, a region which has been described in previous studies to be amplified in several types of cancers [27,28]. As one of the highly conserved components in mammals, YAP has been proved to be a nuclear effector of the Hippo pathway and was initially identified by mosaic screens in Drosophila melanogaster as a vital growth regulator of 


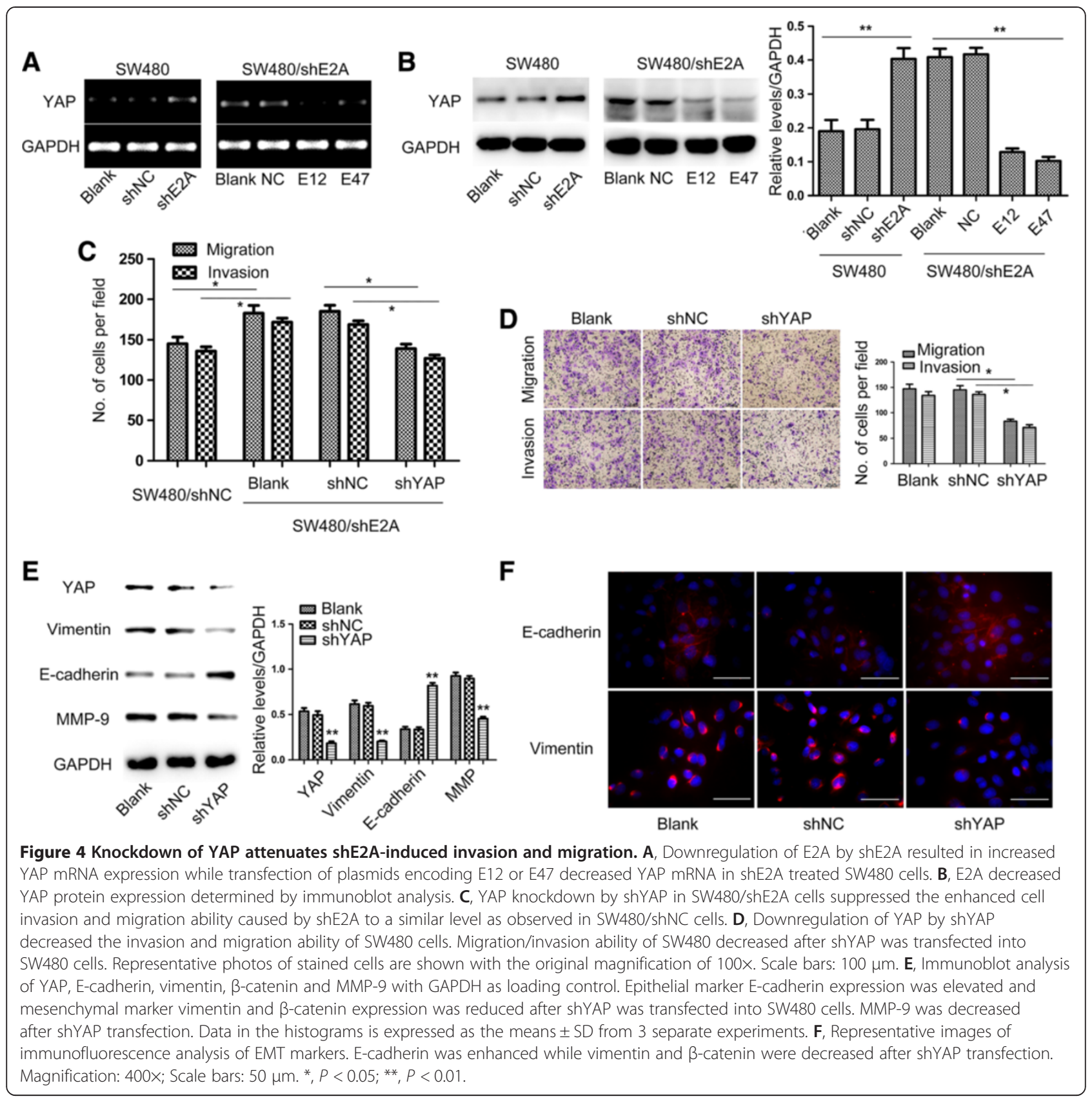

cell proliferation and apoptosis [29-31]. YAP is also a transcriptional modulator which has been implicated in stem cell differentiation, control of organ size, and tumor growth [32,33]. colonic adenocarcinoma tissues show up-regulated YAP expression compared with normal colon tissues, and inducible transgenic expression of a stabilized YAP mutant (S127A) in mice induced colonic adenomas [34,35]. Indeed, Wang et al. found that YAP was a prognostic marker of CRC and down-regulation of YAP reduced the metastatic ability of CRC cells [36]. In our study, we found YAP was inversely associated with $\mathrm{E} 2 \mathrm{~A}$ in $\mathrm{CRC}$ tissues. This further led us to discover that YAP was a downstream target of E2A as its expression was increased upon shE2A transfection while E12 and E47 transfection could reduce it to normal level. Moreover, $\beta$-catenin, which was regulated by E2A, could enhance YAP expression by directly binding to YAP gene in CRC cells [37]. In the present study, we found YAP exerted its function of enhancing metastasis by inducing EMT in CRC cells, which was in consistent with the work of Wang et al. [36]. Importantly, knockdown of YAP in shE2A treated SW480 cells could abolish the elevated cell invasion and migration caused by shE2A. This finding suggested the role of YAP 
in the E2A regulated inhibition of cell invasion and migration. Hence, YAP plays as a downstream in mediating E2A's function as a tumor suppressive gene in CRC.

\section{Conclusion}

The findings of our study suggest that E2A expression is associated with CRC metastasis. By targeting YAP, E2A inhibits EMT program and suppresses invasion and migration in CRC cells. Although E2A's function in cancer has not been fully understood, our findings provide new molecular target and mechanism of action of E2A in CRC metastasis. Therefore, E2A has the potential value to be developed as a new target for CRC prevention and therapy.

\section{Additional files}

Additional file 1: Figure S1. E2A suppresses Caco-2 invasion and migration. A, shE2A expression suppresses invasion and migration of Caco-2 cells. B, E12 or E47 expression decreased cell invasion and migration of Caco-2/shE2A cells. Data represents the means \pm SD from 3 independent experiments. Representative photos of stained cells are shown with the original magnification of $100 \times$. Scale bars: $100 \mu \mathrm{m} .{ }^{*}, P<0.05$.

Additional file 2: Figure S2. Significant inverse correlation between YAP mRNA expression and E2A mRNA expression (Pearson's correlation $R=-0.491, P<0.01)$.

Additional file 3: Figure S3. shYAP decreased the expression of YAP protein in SW480/shE2A cells. Upper penal: Immunoblot analysis using anti-YAP antibodies as indicated with or without shYAP expression. GAPDH was used as loading control. Lower panel: Densitometric analysis of upper panel normalized to GAPDH.

\section{Competing interests}

The authors declare that they have no competing interests.

\section{Authors' contributions}

$\mathrm{HCZ}, \mathrm{AH}$ and PL carried out the experimental studies, drafted the graphs, performed the statistical analysis and wrote the paper. BF and YJQ provided experimental technical supports. ZHM, ZGZ, XHC and MHZ contributed to the study design and manuscript revision, and provided funding for this study. All authors read and approved the final manuscript.

\section{Acknowledgements}

This study was supported by grants from National Natural Science Foundation of China (NSFC, Grant No. 30873000, 81300290 and 81372187).

Received: 12 September 2013 Accepted: 25 November 2013 Published: 26 December 2013

\section{References}

1. Jemal A, Bray F, Center MM, Ferlay J, Ward E, Forman D: Global cancer statistics. CA Cancer J Clin 2011, 61:69-90.

2. Fidler IJ: The pathogenesis of cancer metastasis: the 'seed and soil' hypothesis revisited. Nat Rev Cancer 2003, 3:453-458.

3. Nadal C, Maurel J, Gascon P: Is there a genetic signature for liver metastasis in colorectal cancer? World J Gastroenterol 2007, 13:5832-5844.

4. Murre C, McCaw PS, Baltimore D: A new DNA binding and dimerization motif in immunoglobulin enhancer binding, daughterless, MyoD, and myc proteins. Cell 1989, 56:777-783.

5. Chakraborty T, Brennan TJ, Li L, Edmondson D, Olson EN: Inefficient homooligomerization contributes to the dependence of myogenin on E2A products for efficient DNA binding. Mol Cell Biol 1991, 11:3633-3641.

6. Lassar AB, Buskin JN, Lockshon D, Davis RL, Apone S, Hauschka SD, Weintraub $H$ : MyoD is a sequence-specific DNA binding protein requiring a region of myc homology to bind to the muscle creatine kinase enhancer. Cell 1989, 58:823-831.

7. Murre C, McCaw PS, Vaessin H, Caudy M, Jan LY, Jan YN, Cabrera CV, Buskin JN, Hauschka SD, Lassar AB, et al: Interactions between heterologous helix-loop-helix proteins generate complexes that bind specifically to a common DNA sequence. Cell 1989, 58:537-544.

8. Aronheim A, Shiran R, Rosen A, Walker MD: Cell-specific expression of helix-loop-helix transcription factors encoded by the E2A gene. Nucleic Acids Res 1993, 21:1601-1606.

9. Watada H, Kajimoto $Y$, Umayahara $Y$, Matsuoka T, Morishima T, Yamasaki Y, Kawamori R, Kamada T: Ubiquitous, but variable, expression of two alternatively spliced mRNAs encoding mouse homologues of transcription factors E47 and E12. Gene 1995, 153:255-259.

10. Zhuang $Y$, Soriano $P$, Weintraub $H$ : The helix-loop-helix gene E2A is required for B cell formation. Cell 1994, 79:875-884.

11. LeBrun DP: E2A basic helix-loop-helix transcription factors in human leukemia. Front Biosci 2003, 8:s206-s222.

12. Bain G, Maandag EC, Izon DJ, Amsen D, Kruisbeek AM, Weintraub BC, Krop I, Schlissel MS, Feeney AJ, van Roon M, et al: E2A proteins are required for proper B cell development and initiation of immunoglobulin gene rearrangements. Cell 1994, 79:885-892.

13. Engel I, Murre C: Ectopic expression of E47 or E12 promotes the death of E2A-deficient lymphomas. Proc Natl Acad Sci USA 1999, 96:996-1001.

14. Perez-Moreno MA, Locascio A, Rodrigo I, Dhondt G, Portillo F, Nieto MA, Cano A: A new role for E12/E47 in the repression of E-cadherin expression and epithelial-mesenchymal transitions. J Biol Chem 2001, 276:27424-27431.

15. Wilson JW, Deed RW, Inoue T, Balzi M, Becciolini A, Faraoni P, Potten CS, Norton JD: Expression of Id helix-loop-helix proteins in colorectal adenocarcinoma correlates with p53 expression and mitotic index. Cancer Res 2001, 61:8803-8810.

16. Li P, Lin Y, Zhang Y, Zhu Z, Huo K: SSX2IP promotes metastasis and chemotherapeutic resistance of hepatocellular carcinoma. J Trans/ Med 2013, 11:52

17. Li P, Chen X, Su L, Li C, Zhi Q, Yu B, Sheng H, Wang J, Feng R, Cai Q, et al: Epigenetic silencing of miR-338-3p contributes to tumorigenicity in gastric cancer by targeting SSX2IP. PLOS One 2013, 8:e66782.

18. Polyak K, Weinberg RA: Transitions between epithelial and mesenchymal states: acquisition of malignant and stem cell traits. Nat Rev Cancer 2009, 9:265-273.

19. Yang J, Weinberg RA: Epithelial-mesenchymal transition: at the crossroads of development and tumor metastasis. Dev Cell 2008, 14:818-829.

20. Thiery JP: Epithelial-mesenchymal transitions in tumour progression. Nat Rev Cancer 2002, 2:442-454.

21. Markowitz SD, Bertagnolli MM: Molecular origins of cancer: molecular basis of colorectal cancer. N Engl J Med 2009, 361:2449-2460.

22. Gupta GP, Massague J: Cancer metastasis: building a framework. Cell 2006, 127:679-695.

23. Hwang-Verslues WW, Chang PH, Wei PC, Yang CY, Huang CK, Kuo WH, Shew JY, Chang KJ, Lee EY, Lee WH: miR-495 is upregulated by E12/E47 in breast cancer stem cells, and promotes oncogenesis and hypoxia resistance via downregulation of E-cadherin and REDD1. Oncogene 2011, 30:2463-2474

24. Hata F, Nishimori H, Yasoshima T, Tanaka H, Ohno K, Yanai Y, Ezoe E, Kamiguchi $\mathrm{K}$, Isomura $\mathrm{H}$, Denno R, et al: Profiling analysis of differential gene expression between hematogenous and peritoneal metastatic sublines of human pancreatic cancer using a DNA chip. J Exp Clin Cancer Res 2004, 23:513-520.

25. Thiery JP, Acloque $H$, Huang RY, Nieto MA: Epithelial-mesenchymal transitions in development and disease. Cell 2009, 139:871-890.

26. Oh SJ, Shin JH, Kim TH, Lee HS, Yoo JY, Ahn JY, Broaddus RR, Taketo MM, Lydon JP, Leach RE, et al: beta-catenin activation contributes to the pathogenesis of adenomyosis through epithelial-mesenchymal transition. J Pathol 2013, 231:210-222.

27. Overholtzer M, Zhang J, Smolen GA, Muir B, Li W, Sgroi DC, Deng CX, Brugge JS, Haber DA: Transforming properties of YAP, a candidate oncogene on the chromosome 11q22 amplicon. Proc Natl Acad Sci U S A 2006, 103:12405-12410.

28. Zender L, Spector MS, Xue W, Flemming P, Cordon-Cardo C, Silke J, Fan ST, Luk JM, Wigler M, Hannon GJ, et al: Identification and validation of 
oncogenes in liver cancer using an integrative oncogenomic approach. Cell 2006, 125:1253-1267.

29. Dong J, Feldmann G, Huang J, Wu S, Zhang N, Comerford SA, Gayyed MF, Anders RA, Maitra A, Pan D: Elucidation of a universal size-control mechanism in Drosophila and mammals. Cell 2007, 130:1120-1133.

30. Pan D: Hippo signaling in organ size control. Genes Dev 2007, 21:886-897.

31. Edgar BA: From cell structure to transcription: hippo forges a new path. Cell 2006, 124:267-273.

32. Pan D: The hippo signaling pathway in development and cancer. Dev Cell 2010, 19:491-505.

33. Zhao B, Ye X, Yu J, Li L, Li W, Li S, Lin JD, Wang CY, Chinnaiyan AM, Lai ZC, Guan KL: TEAD mediates YAP-dependent gene induction and growth control. Genes Dev 2008, 22:1962-1971.

34. Steinhardt AA, Gayyed MF, Klein AP, Dong J, Maitra A, Pan D, Montgomery EA, Anders RA: Expression of Yes-associated protein in common solid tumors. Hum Pathol 2008, 39:1582-1589.

35. Camargo FD, Gokhale S, Johnnidis JB, Fu D, Bell GW, Jaenisch R, Brummelkamp TR: YAP1 increases organ size and expands undifferentiated progenitor cells. Curr Biol 2007, 17:2054-2060

36. Wang L, Shi S, Guo Z, Zhang X, Han S, Yang A, Wen W, Zhu Q: Overexpression of YAP and TAZ is an independent predictor of prognosis in colorectal cancer and related to the proliferation and metastasis of colon cancer cells. PLoS One 2013, 8:e65539.

37. Konsavage WM Jr, Kyler SL, Rennoll SA, Jin G, Yochum GS: Wnt/betacatenin signaling regulates Yes-associated protein (YAP) gene expression in colorectal carcinoma cells. J Biol Chem 2012, 287:11730-11739.

doi:10.1186/1479-5876-11-317

Cite this article as: Zhao et al.: E2A suppresses invasion and migration by targeting YAP in colorectal cancer cells. Journal of Translational Medicine 2013 11:317.

\section{Submit your next manuscript to BioMed Central and take full advantage of:}

- Convenient online submission

- Thorough peer review

- No space constraints or color figure charges

- Immediate publication on acceptance

- Inclusion in PubMed, CAS, Scopus and Google Scholar

- Research which is freely available for redistribution 